\title{
Estudo teórico da eficiência de inibidores orgânicos de corrosão derivados do benzimidazol
}

DOI: $10.30609 / J E T I .2018-1.5270$

\section{Frederico M. da Silva ${ }^{1 *}$, Lillian W. C. Paes ${ }^{1}$}

${ }^{1}$ UFF/EEIMVR/PPGEM, Volta Redonda-RJ

\section{Resumo}

O objetivo deste trabalho foi avaliar através de cálculos quânticos a eficiência de inibição frente à corrosão de duas moléculas orgânicas derivadas do benzimidazol, mais especificamente, o 2mercaptobenzimidazol (2Mcb) e 2-fenilbenzimidazol (2Fb). Os cálculos foram realizados utilizando o método da Teoria do Funcional de Densidade (TFD), com o funcional B3LYP e com conjunto de funções de base 6$311+G(d, p)$. Os parâmetros quânticos correlacionados com a eficiência de

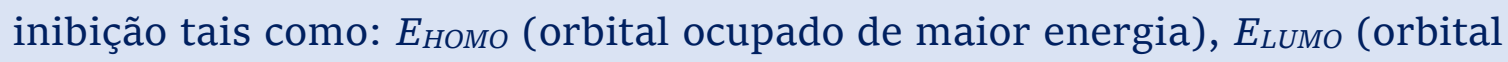
molecular desocupado de menor energia), diferença de energia $(\Delta E)$, eletronegatividade $(\chi)$, dureza $(\eta)$, fração de elétrons transferidos $(\Delta N)$, eletrofilicidade $(\omega)$ e índices de Fukui, foram calculados. Também foram realizados cálculos em meio aquoso nas formas protonadas e não protonadas. Os resultados teóricos foram comparados com dados experimentais e foi verificada uma boa correlação entre os parâmetros químico-quânticos e eficiência de inibição das moléculas.

Key words: benzimidazol, TFD, moléculas orgânicas, corrosão. 


\section{1.- Introdução}

A corrosão de materiais recebe uma atenção considerável como resultado da sua relevância industrial. 0 uso de inibidores de corrosão é provavelmente o método mais viável do ponto de vista econômico e facilidade de aplicação [1]. Dentre inúmeras iniciativas de inibidores de corrosão, a seleção de moléculas orgânicas como inibidores vem sendo amplamente utilizadas em vista de sua vantagem econômica, maior eficiência e viabilidade tecnológica.

Os inibidores orgânicos provocam a formação de um filme passivador na superfície metálica. A eficiência de inibição tem sido relatada por vários autores [2-5] segundo a composição química, a estrutura espacial molecular, a estrutura eletrônica molecular, a densidade de carga superficial e sua afinidade com a superfície do metal.

Vários compostos orgânicos que contêm heteroátomos tais como: nitrogênio, oxigênio, fósforo, enxofre, anéis aromáticos e/ou ligações $\pi$ de elétrons têm sido relatados como inibidores orgânicos de corrosão de metais em diferentes meios agressivos [6-10]. Além disso, existe uma interação específica entre grupos substituintes e o metal, os heteroátomos e os pares de elétrons livres são importantes características que determinam a adsorção das moléculas na superfície metálica. A geometria de um inibidor também influencia sua adsorção na interface metálica. Moléculas mais planas têm maior tendência de se adsorverem em relação às que possuem menor planaridade geométrica [11].

Deve-se ressaltar que pesquisas no campo dos inibidores de corrosão nos últimos anos têm sido direcionadas para a concepção e busca de inibidores "verdes" devido às novas regulamentações ambientais para a substituição dos produtos tóxicos [12-15].

O benzimidazol é um composto orgânico e heterocíclico, apresentando um anel aromático e outro imidazólico. Essa molécula e seus derivados estão entre os compostos, que inibem a corrosão do ferro em solução aquosa de $\mathrm{HCl}$, vários estudos sobre a eficiência de inibição à corrosão do aço-C (baixo teor de C) relacionando os derivados do benzimidazol têm sido relatados na literatura [16$18]$. 
Assim, neste artigo foram avaliados os parâmetros quânticos das moléculas 2-mercaptobenzimidazol (2Mcb) e 2-fenilbenzimidazol (2Fb), frente a inibição de corrosão, além de encontrar uma correlação entre as e estruturas eletrônicas dos inibidores e a eficiência de inibição.

\section{2.- Detalhes Computacionais}

Os cálculos computacionais foram realizados utilizando o programa Gaussian 09w [19] através do método da Teoria do Funcional da Densidade (TFD) com o funcional híbrido $B 3 L Y P$ aliado ao conjunto de bases $6-311+G(d, p)$. A análise de frequência vibracional foi realizada para garantir que todas as moléculas derivadas do benzimidazol atingissem seus respectivos estados fundamentais, comprovando os mínimos globais. Geralmente, o fenômeno de corrosão eletroquímica ocorre em meio aquoso, desta forma, faz-se necessário considerar o efeito do solvente nos cálculos. Assim, o método de solvatação CPCM (Polarizable Conductor Calculation Model) foi aplicado para incorporar o efeito do solvente $\left(\mathrm{H}_{2} \mathrm{O}\right)$ no cálculo $T F D$.

Neste artigo foram calculadas as propriedades quânticas tais como: as energias dos orbitais de fronteira (HOMO e $L U M O)$, a diferença de energia $(\Delta E)$, afinidade eletrônica $(A)$, energia de ionização $(I)$, eletronegatividade $(\chi)$, dureza $(\eta)$, fração de elétrons transferidos $(\Delta N)$ e eletrofilicidade $(\omega)$.

De acordo com o teorema de Koopman [20], o potencial de ionização (I), afinidade eletrônica $(A)$, a eletronegatividade $(\chi)$ e a dureza $(\eta)$, podem ser definidos em termos da energia do HOMO e do LUMO.

A energia de ionização $(I)$ pode ser aproximada ao valor negativo da energia correspondente ao orbital molecular ocupado de mais alta energia (HOMO).

$$
I=-E_{\text {Номо }}
$$

O orbital molecular desocupado de menor energia (LUMO) está relacionado de forma semelhante à afinidade eletrônica $(A)$. 


$$
A=-E_{L U M O}
$$

A diferença de energia $H O M O$ e $L U M O$, denominada GAP de energia $(\triangle E)$, essa grandeza se mostra mais relevante devido à relação entre os orbitais de fronteira.

$$
\Delta E=E_{\text {LUMO }}-E_{\text {НОМO }}
$$

A eletronegatividade $(\chi)$ e a dureza $(\eta)$ são avaliadas com base na aproximação de diferenças finitas, como combinações lineares de $I$ e $A$ calculados [21], onde $I$ e $A$ são potencial de ionização e afinidade eletrônica, respectivamente.

$$
\begin{aligned}
& \chi=\frac{I+A}{2} \\
& \eta=\frac{I-A}{2}
\end{aligned}
$$

Para calcular a fração de elétrons transferidos $(\Delta N)$, é necessário utilizar os valores teóricos de eletronegatividade e de dureza do ferro ( $\mathrm{Fe}$ ), $\chi_{F e}=7,0 \mathrm{eV}$ e $\eta_{F e}=0$ $\mathrm{eV}$, respectivamente [22].

$$
\Delta N=\frac{\chi_{F e}-\chi_{i n i b}}{2\left(\eta_{F e}+\eta_{i n i b}\right)}
$$

0 índice de eletrofilicidade $(\omega)$ se refere somente aos valores de eletronegatividade e dureza das moléculas.

$$
\omega=\frac{\chi^{2}}{2 \eta}
$$

0 índice de Fukui é um parâmetro associado à reatividade de um determinado sítio de adsorção na molécula, e fornece informações úteis na identificação do comportamento eletrofílico/nucleofílico. As funções de Fukui foram calculadas utilizando o programa UCA-FUKUI_V2 [23] uma vez que fornece uma boa análise da seletividade local de um inibidor de corrosão. As expressões a 
seguir são derivadas da densidade eletrônica em relação ao número de elétrons com um potencial externo constante $[24,25]$.

$$
\begin{gathered}
f_{k}^{+}=q_{k}(N+1)-q_{k}(N) \text { (reação nucleofílica) } \\
f_{k}^{-}=q_{k}(N)-q_{k}(N-1)(\text { reação eletrofílica) }
\end{gathered}
$$

onde $q_{k}$ representa a carga elétrica do átomo $k, q_{k}(N+1), q_{k}(N)$ e $q_{k}(N-1)$ são definidas como cargas atômicas das espécies aniônicas, neutras e catiônicas, respectivamente.

\section{3.- Resultados e Discussões}

Os parâmetros estruturais dos inibidores 2-mercaptobenzimidazol (2Mcb) e 2-fenilbenzimidazol ( $2 \mathrm{Fb}$ ) foram calculados em fase gasosa e comparados com os dados experimentais da estrutura cristalina de raios-X dos derivados do benzimidazol [26], onde foi observado uma boa concordância com os dados experimentais. Deve-se ressaltar que não ocorreram mudanças significativas nos parâmetros estruturais quando comparados com os cálculos utilizando o solvente. A figura 1 apresenta a estrutura das moléculas otimizadas com solvente e não protonadas. Nota-se que a protonação não modificou os parâmetros estruturais dos derivados de benzimidazol.

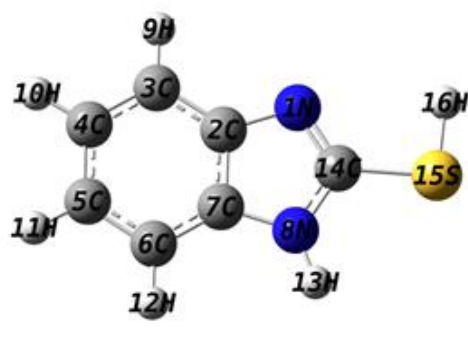

(a)

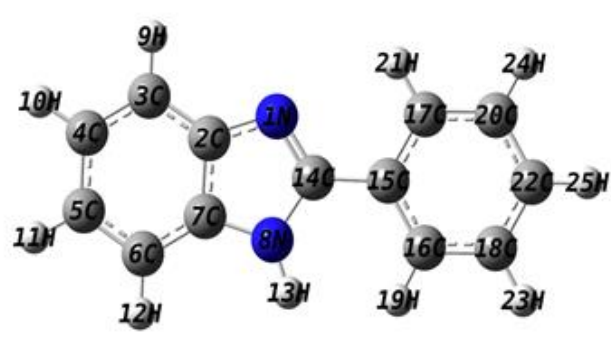

(b)

Figura 1: Estrutura otimizada do 2-mercaptobenzimidazol (a) e do 2fenilbenzimidazol (2) com o conjunto $B 3 L Y P / 6-311+G(d, p)$. 
A figura 2 apresenta a distribuição das densidades eletrônicas nos orbitais de fronteira $H O M O / L U M O$ dos derivados de benzimidazol com solvente $\left(\mathrm{H}_{2} \mathrm{O}\right)$.

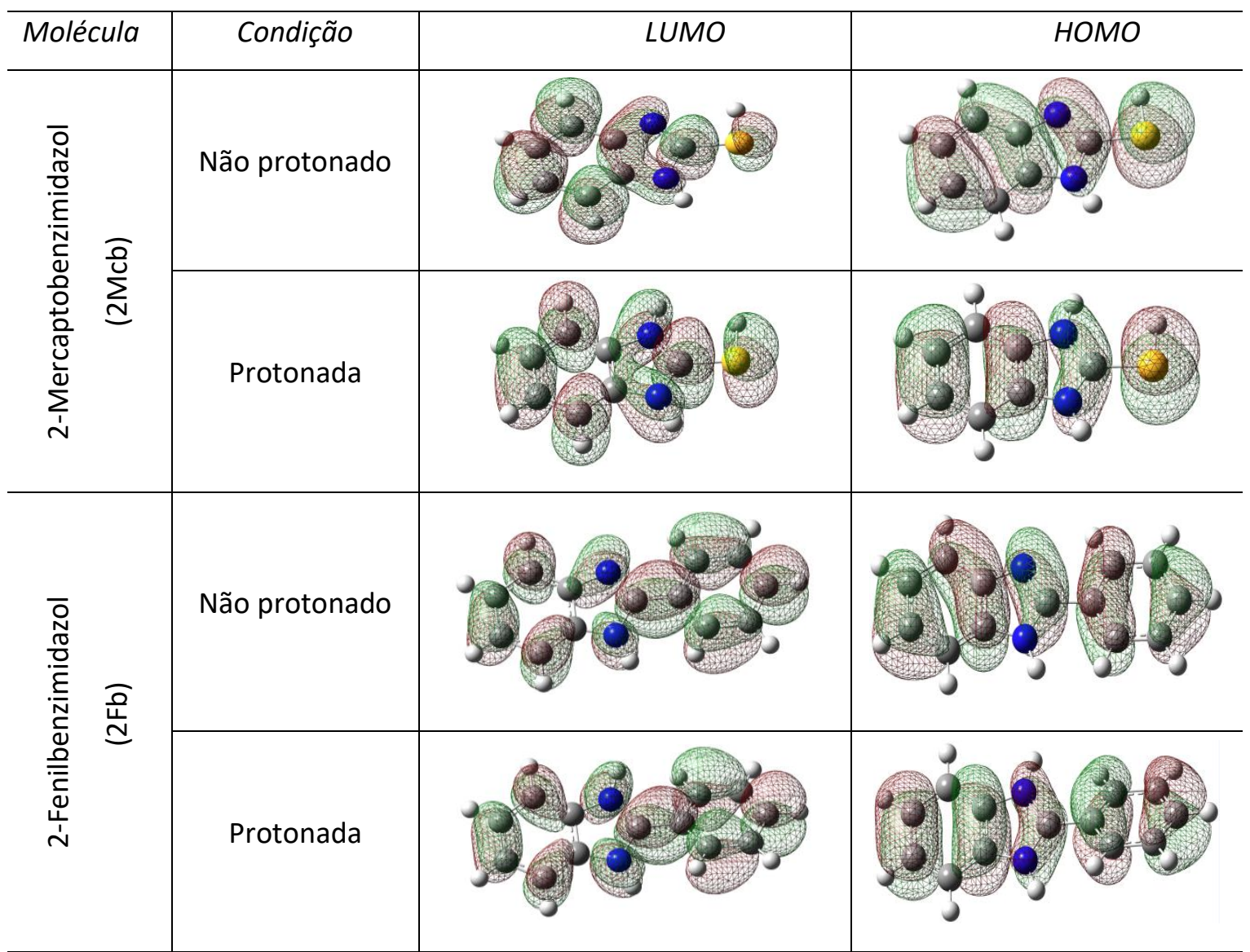

Figura 2: Diagramas de contorno dos orbitais de fronteira calculados com o conjunto $B 3 L Y P / 6-311+G(d, p)$ com solvente $\left(\mathrm{H}_{2} \mathrm{O}\right)$.

Analisando a figura 2, pode-se observar que o HOMO para as moléculas protonadas e não-protonadas mostram que esse orbital é deslocalizado sobre toda a molécula, sendo de caráter $\pi$ ligante. 0 HOMO está em grande parte localizado no anel de benzeno e no anel de imidazol. A distribuição HOMO é planar fazendo com que a molécula seja adsorvida perfeitamente na superfície metálica. Observa-se que os átomos C3 e C6 não contribuem para formação dos HOMOs das duas moléculas protonadas. Os LUMOs apresentam um caráter $\pi$ antiligante.

A tabela 1 apresenta os valores dos parâmetros químico-quânticos calculados com o funcional $B 3 L Y P$ e a base $6-311+G(d, p)$ para as moléculas em solução. 
Tabela 1: Parâmetros químico-quânticos calculados com o conjunto $B 3 L Y P / 6-311+G(d, p)$ com solvente $\left(\mathrm{H}_{2} \mathrm{O}\right)$.

\begin{tabular}{|c|c|c|c|c|}
\hline \multirow{2}{*}{ Parâmetros teóricos } & \multicolumn{2}{|c|}{ 2-Mercaptobenzimidazol } & \multicolumn{2}{|c|}{ 2-Fenilbenzimidazol } \\
\hline & Não protonada & Protonada & Não protonada & Protonada \\
\hline $\begin{array}{c}\text { Energia absoluta } \\
\qquad\left(\Delta H_{f}\right),[\text { u.a.] }\end{array}$ & $-778,19$ & $-778,64$ & $-611,10$ & $-611,54$ \\
\hline$L U M O,[e V]$ & $-0,94$ & $-1,94$ & $-1,73$ & $-2,57$ \\
\hline HOMO, $[\mathrm{eV}]$ & $-6,25$ & 7,20 & $-6,23$ & $-7,17$ \\
\hline $\begin{array}{c}\text { GAP de energia } \\
(\Delta E),[\mathrm{eV}]\end{array}$ & 5,31 & 5,26 & 4,50 & 4,60 \\
\hline $\begin{array}{l}\text { Eletronegatividade } \\
\qquad(\chi),[\mathrm{eV}]\end{array}$ & 3,60 & 4,57 & 3,98 & 4,87 \\
\hline Dureza $(\eta),[e V]$ & 2,66 & 2,63 & 2,25 & 2,30 \\
\hline $\begin{array}{c}\text { Fração de elétrons } \\
\text { transferidos }(\Delta N)\end{array}$ & 0,64 & 0,46 & 0,67 & 0,46 \\
\hline $\begin{array}{c}\text { Eletrofilicidade } \\
\qquad(\omega),[\mathrm{eV}]\end{array}$ & 2,43 & 3,97 & 3,51 & 5,15 \\
\hline
\end{tabular}

O orbital molecular ocupado de maior energia (HOMO), também conhecido por potencial de ionização $(I)$, tem como característica principal uma relação com a doação de elétrons para o orbital-d do substrato metálico. Portanto, as moléculas que apresentam maior valor de energia HOMO tendem apresentar melhores condições para fornecer elétrons ao orbital-d desocupado do metal e consequentemente terão melhor eficiência de inibição [27]. Os valores de energia dos HOMOs tanto para forma protonada quanto para forma não protonada foram próximos entre si, observa-se que a molécula 2-fenilbenzimidazol (2Fb) apresentou um valor ligeiramente maior.

O orbital molecular desocupado de menor energia (LUMO), também conhecido como afinidade eletrônica $(A)$, revela a tendência das moléculas receberem elétrons provenientes de espécies ricas em elétrons. Valores mais baixos de energia LUMO correspondem a maior capacidade de receber elétrons da superfície metálica e maior eficiência de inibição da molécula [28]. Verificou-se que para os valores de energia do LUMO da molécula 2-fenilbenzimidazol (2Fb), 
apresentaram valores inferiores aos encontrados para a molécula 2mercaptobenzimidazol (2Mcb).

A diferença de energia HOMO/LUMO $(\Delta E)$ demonstra uma dos mais relevantes parâmetros, devido à relação entre os orbitais de fronteira [29]. A diferença de energia tem uma relação intrínseca com a reatividade química da molécula, ou seja, moléculas mais reativas possuem menores valores de $\Delta E$, porque a energia necessária para remover um elétron do orbital ocupado de mais baixa energia será menor, e consequentemente têm maior desempenho quanto à inibição [30]. Os valores $\Delta E$ seguiram as mesmas tendências quando analisadas as formas protonadas e não protonadas. Observa-se que a molécula 2fenilbenzimidazol $(2 \mathrm{Fb})$ apresentou o menor valor de $\Delta E$.

A eletronegatividade $(\chi)$ indica a capacidade molecular em aceitar elétrons, moléculas e substratos possuem diferentes valores dessa grandeza. 0 princípio de Sanderson [31] afirma que dois ou mais átomos se unem para formar uma molécula e suas eletronegatividades se ajustam para um valor intermediário. Uma tendência descrita na literatura relata que as menores diferenças entre as eletronegatividades (inibidor e metal) resultam no equilíbrio atingido mais rapidamente, promove menor reatividade e consequentemente a eficiência de inibição será reduzida [32]. Os valores de eletronegatividade da molécula 2mercaptobenzimidazol (2Mcb) foram inferiores, em ambas as condições, aos valores do 2-fenilbenzimidazol (2Fb).

A relação entre a dureza $(\eta)$ e a diferença de energia $(\Delta E)$ é fisicamente clara. É descrito na literatura que a estrutura molecular mais estável possui maior valor de $\Delta E$ [33]. Por outro lado, o sistema eletrônico com maior $\Delta E$ deve ser menos reativo quimicamente. Essa relação se baseia na teoria HSAB (Hard and Soft Acid-Base) de Pearson [34]. Observa-se novamente a mesma tendência para as espécies protonadas e não protonadas.

A fração de elétrons transferidos $(\Delta N)$, segundo Pearson [35], trata-se dos elétrons que fluem da molécula do inibidor para a superfície metálica. Quando uma reação química contém duas espécies com diferentes eletronegatividades, o seguinte mecanismo é verificado: os elétrons fluem da espécie que possui menor 
eletronegatividade (inibidor) em direção à espécie de maior eletronegatividade (superfície metálica) até que o equilíbrio entre os potenciais químicos seja atingido. 0 valor teórico da eletronegatividade do ferro é $\chi_{F e}=7,0 \mathrm{eV}$ [22] e a dureza desse metal comumente utilizada para esse cálculo é $\eta_{F e}=0 \mathrm{eV}$. De acordo com Lukovits [36], se $\Delta N<3,6$; a eficiência de inibição da molécula orgânica cresce com o aumento da habilidade de eletrodoação na superfície metálica. Os valores de $\Delta N$ das moléculas foram iguais quando comparados na forma protonada, mas o 2fenilbenzimidazol (2Fb) obteve maiores valores na forma não protonada.

0 índice de eletrofilicidade $(\omega)$ mede a capacidade de uma espécie química em aceitar elétrons, assim, a molécula de 2-fenilbenzimidazol ( $2 \mathrm{Fb}$ ), possui a maior capacidade de aceitar elétrons, o que está de acordo com a tendência observada nas energias LUMOs.

A partir de dados experimentais encontrados na literatura [17,37], foi possível traçar um perfil gráfico comparando o desempenho de inibição entre as duas moléculas, conforme a figura 3.

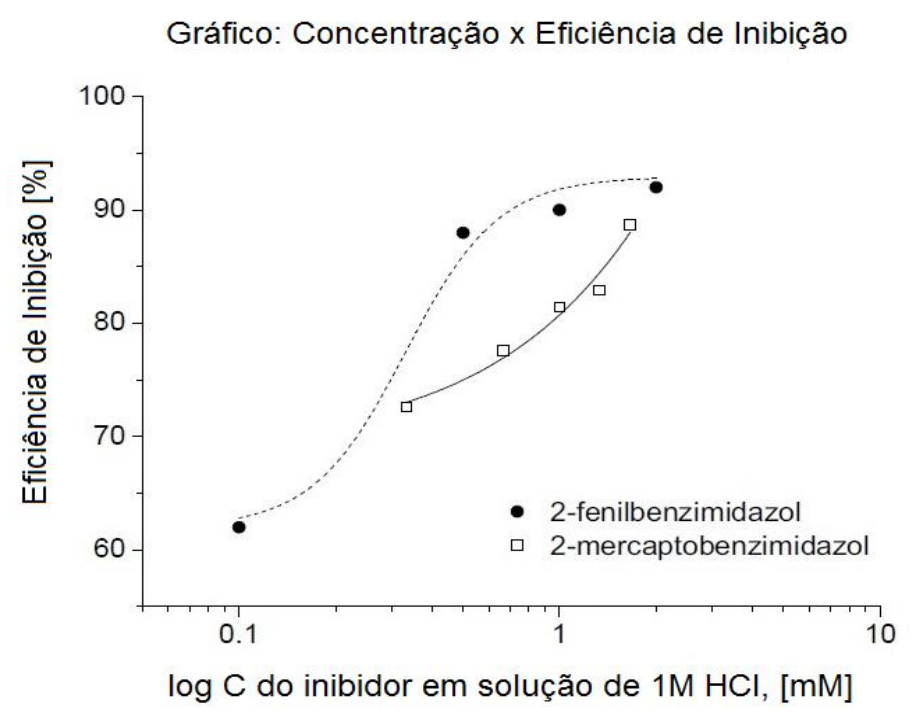

Figura 3: Gráfico de desempenho das moléculas protonadas obtido através de curvas de polarização sob um aço-C (baixo teor de C).

O gráfico da figura 3 evidenciou resultados das curvas de polarização sobre um aço-C (baixo teor de carbono) onde a maior eficiência de inibição foi obtida 
segundo a ordem: $2 \mathrm{Fb}>2 \mathrm{Mcb}$, em todas as concentrações coincidentes. Deve-se considerar a concentração de $1 \mathrm{M} \mathrm{HCl}$ na solução dos ensaios, devido ao fato dessa concentração ser suficientemente alta para que as moléculas atinjam o estado protonado quando adicionadas ao meio.

$\mathrm{Na}$ tabela 2, encontram-se os valores dos índices de Fukui calculados considerando as moléculas como não protonadas em meio aquoso. 0 funcional utilizado para os cálculos foi o $B 3 L Y P$ e a base $6-311+G(d, p)$.

Tabela 2: Índices de Fukui calculados com o conjunto $B 3 L Y P / 6-311+G(d, p)$. Os valores foram obtidos considerando as moléculas não protonadas com solvente $\left(\mathrm{H}_{2} \mathrm{O}\right)$.

\begin{tabular}{c|c|c|c|c|c}
\hline \multicolumn{2}{c|}{ 2-Mercaptobenzimidazol (2Mcb) } & \multicolumn{3}{c}{ 2-Fenilbenzimidazol (2Fb) } \\
\hline Átomo & $f_{k}{ }^{-}$ & $f_{k}{ }^{+}$ & Átomo & $f_{k}^{-}$ & $f_{k}{ }^{-}$ \\
\hline N1 & 0,0809 & 0,0532 & N1 & 0,0728 & 0,0652 \\
C2 & 0,0776 & 0,0505 & C2 & 0,0712 & 0,0220 \\
C3 & 0,0570 & $\mathbf{0 , 1 2 3 6}$ & C3 & 0,0540 & 0,0501 \\
C4 & 0,0645 & 0,0697 & C4 & 0,0573 & 0,0336 \\
C5 & 0,1028 & 0,0912 & C5 & $\mathbf{0 , 0 9 5 5}$ & 0,0464 \\
C6 & 0,0446 & $\mathbf{0 , 1 2 1 0}$ & C6 & 0,0417 & 0,0441 \\
C7 & 0,0689 & 0,0379 & C7 & 0,0635 & 0,0199 \\
N8 & 0,0318 & 0,0322 & N8 & 0,0281 & 0,0345 \\
H9 & 0,0295 & 0,0588 & H9 & 0,0276 & 0,0230 \\
H10 & 0,0301 & 0,0359 & H10 & 0,0274 & 0,0172 \\
H11 & 0,0378 & 0,0441 & H11 & 0,0351 & 0,0214 \\
H12 & 0,0259 & 0,0562 & H12 & 0,0242 & 0,0200 \\
H13 & 0,0225 & 0,0221 & H13 & 0,0194 & 0,0201 \\
C14 & 0,0617 & 0,0809 & C14 & 0,0606 & 0,0678 \\
S15 & $\mathbf{0 , 2 2 7 7}$ & 0,1019 & C15 & 0,0355 & 0,0631 \\
H16 & 0,0368 & 0,0206 & C16 & 0,0382 & 0,0609 \\
& & & C17 & 0,0415 & 0,0609 \\
& & & C18 & 0,0307 & 0,0466 \\
& & & H19 & 0,0166 & 0,0290 \\
& & & C20 & 0,0273 & 0,0477 \\
& & & H21 & 0,0194 & 0,0319 \\
& & & H23 24 & 0,0590 & $\mathbf{0 , 0 8 7 1}$ \\
& & & 0,0162 & 0,0245 \\
& & & 0,0155 & 0,0249 \\
& & & 0,0219 & 0,0381 \\
\hline
\end{tabular}

As moléculas inibidoras podem se ligar à superfície metálica através de transferência de elétrons (doação ou aceitação). Portanto, em um inibidor é 
essencial examinar os sítios ativos de interação. Uma análise dos índices de Fukui permite determinar a tendência de sítios preferenciais de adsorção através de reações eletrofílicas ou nucleofílicas. Quanto maior forem os índices $f_{k}{ }^{+}$e $f_{k}$, maior serão as evidências de sítios nucleofílico e eletrofílico, respectivamente. 0 valor de $f_{k}{ }^{+}$mede as mudanças na densidade eletrônica quando a molécula aceita elétrons adicionais, enquanto a densidade de elétrons medida em $f_{k}{ }^{+}$representa a mudança quando a molécula perde elétrons.

Uma análise dos índices de Fukui mostrados na tabela 2 é possível observar que o átomo S15 $\left(f_{k^{-}}=0,2277\right)$ da molécula 2-mercaptobenzimidazol $(2 \mathrm{Mcb})$ é um sítio mais susceptível para o ataque eletrofílico, o que indica que os elétrons pertencentes ao S15 são mais capazes de interagir com a superfície metálica (cátions do metal). Enquanto os átomos C3 $\left(f_{k^{+}}=0,1236\right)$ e C6 $\left(f_{k^{+}}=0,1210\right)$ são sítios mais susceptíveis para o ataque nucleofílico.

Analisando para a molécula 2-fenilbenzimidazol (2Fb), observa-se que o átomo C5 $\left(f_{k^{-}}=0,0955\right)$, é um possível sítio para o ataque eletrofílico, sendo este situado sobre o anel benzênico. 0 átomo C22 $\left(f_{k^{+}}=0,0871\right)$ apresenta um sítio para o ataque nucleofílico localizado sobre o grupo substituinte fenil.

A figura 4 apresenta o mapa do potencial eletrostático (MEP) dos derivados do benzimidazol, calculados através do funcional $B 3 L Y P$ e a base $6-311+G(d, p)$ com solvente $\left(\mathrm{H}_{2} \mathrm{O}\right)$. $\mathrm{O}$ mapa do potencial eletrostático (MEP) calculado reflete a distribuição da densidade eletrônica dentro da molécula. Observa-se que o átomo de nitrogênio N1 para os dois derivados apresenta a maior carga negativa (vermelho). É notável a diferença entre as condições não protonada (à esquerda) e protonada (à direita). A figura 4 indica que a polaridade das moléculas não protonadas é superior à condição protonada. Observa-se que quando protonadas as moléculas obtiveram maior uniformidade na distribuição das densidades eletrônicas. 

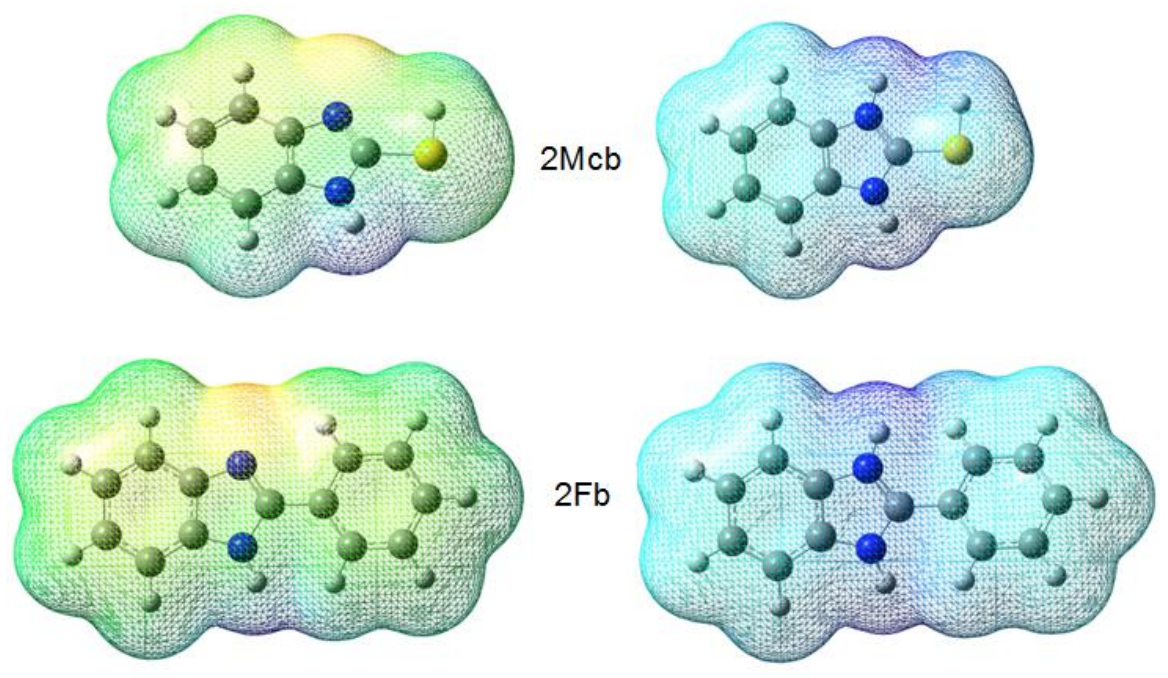

Alta

Baixa

Figura 4: Mapa do potencial eletrostático (MEP) das moléculas 2Mcb e 2Fb não protonadas (à esquerda) e protonadas (à direita).

\section{4.- Conclusões}

Para analisar o comportamento reativo dos derivados de benzimidazol e correlacionar as estruturas eletrônicas com a eficiência de inibição, um estudo teórico foi realizado utilizando o método da Teoria do Funcional de Densidade com funcional $B 3 L Y P$ e a base $6-311+G(d, p)$.

A capacidade de inibição dos dois derivados de benzimidazol estudados mostrou estreita correlação com a maioria dos parâmetros químico-quânticos, tais como: as energias dos orbitais de fronteira (HOMO e LUMO), a diferença de energia $(\Delta E)$, a dureza $(\eta)$ e fração de elétrons transferidos $(\Delta N)$ com os dados experimentais.

Os HOMOs e LUMOs estão localizados no anel benzênico e no anel imidazólico, que revelaram os locais ativos responsáveis pela reação eletrofílica e nucleofílica da molécula inibidora com o aço. Os HOMOs são de caráter $\pi$ ligante, enquanto os LUMOs apresentam um caráter $\pi$ antiligante.

Os índices de Fukui evidenciaram os sítios preferenciais de adsorção das moléculas, o átomo S15 $\left(f_{k^{-}}=0,2277\right)$ e os átomos C3 $\left(f_{k^{+}}=0,1236\right)$ e C6 $\left(f_{k^{+}}=\right.$ 
0,1210) para o derivado 2-mercaptobenzimidazol (2Mcb). Enquanto o átomo $\mathrm{C5}$ $\left(f_{k^{-}}=0,0955\right)$ e o átomo C22 $\left(f_{k^{+}}=0,0871\right)$ para o derivado 2 -fenilbenzimidazol $(2 \mathrm{Fb})$.

Finalmente, pode-se concluir que o efeito da protonação produziu pequenas mudanças na densidade eletrônica dos derivados de benzimidazol, como é observado no mapa de potencial eletrostático.

Com base na análise dos resultados obtidos, através do método da Teoria do Funcional de Densidade, foi possível estabelecer uma ordem de eficiência teórica $2 \mathrm{Fb}>2 \mathrm{Mcb}$, essa ordem encontra-se de acordo com os dados experimentais encontrados na literatura.

Agradecimentos

Os autores desse trabalho são gratos aos órgãos de pesquisa nacional, em especial ao PPGEM/UFF, FAPERJ e CAPES.

\section{5.- Bibliografia}

[1] M.B. VALCARCE, M. VÁZQUEZ, Electrochemica Acta 53, 5007-5015 (2008).

[2] S. A. UMOREN, I. B. OBOT, Surface Review and Letters 15, 277 (2008).

[3] U. ERGUN, D. YUZER, C. EMREGUL, Mater. Chem. Phys. 109, 492-499 (2008).

[4] E.E. Ebenso, H. Alemu, S.A. Umoren, I.B. Obot, Int. J. Electrochem. Sci. 3, 13251339 (2008).

[5] A. YILDIRIM, M. ÇETIN, Corrosion Science 50, 155-165 (2008).

[6] R. HASANOV, M. SADIKOGLU, S. BILGIC, Appl. Surf. Sci. 253, 3913-3921 (2007).

[7] F. BENTISS, M. TRAiSNEL, L. GENGEMBRE, M.A. LAGRENEE, Appl. Surf. Sci. 152, 237-249 (1999).

[8] A. OUCHRIF, M. ZEGMOUT, B. HAMMOUTI, S. EL-KADIRI, A. RAMDANI, Appl. Surf. Sci. 252, 339-334 (2005).

[9] E.S.M. SHERIF, Appl. Surf. Sci. 252, 8615-8623 (2006).

[10] B. LIU, H. XI, Z. LI, Q. XIA, Appl. Surf. Sci. 258, 6679-6687 (2012). 
[11] P. LIU, X. FANG, Y. TANG, C. SUN, C. YAO, Materials Sci. and Appl. 2, 12681272 (2011).

[12] M.E. MASHUGA, L.O. OLASUNKANMI, A.S. ADEKUNLE, S. YESUDASS, M.M. KABANDA, E.E. EBENSO. Materials 8, 3607-3632 (2015).

[13] A.A. SPASOV, I.N. YOZHITSA, L.I. BUGAEVA, V.A. ANISIMOVA, Pharm. Chem. J. 33, 6 (1999).

[14] J. WEBER, M. ANTONIETTI, A. THOMAS, Macromolecules 40, 1299 (2007).

[15] R. RASTOGI, S. SHARMA, Synthesis 861 (1983).

[16] F.B. RAVARI, A. DADGARINEZHAD, I. SHEKHSHOAEI, G. U. Journal of Science 22, 175-182 (2009).

[17] A. POPOVA, M. CHRISTOV, T. DELIGEORIGIEV. Corrosion Science 59, 756764 (2003).

[18] I.B. OBOT, N.O. OBI-EGBEDI, Colloids and Surfaces A: Physicochem. Eng. Aspects 330, 207-212 (2008).

[19] Æ. FRISCH, Gaussian 09W, Gaussian, Inc., Wallingford, CT, 2009.

[20] T. KOOPMAN. Physica 1, 104-113 (1933).

[21] C.G. ZHAN, J.A. NICHOLS, D.A. DIXON. J. Phys. Chem. A 107, 4184-4195 (2003).

[22] V.S. SASTRI, J.R. PERUMAREDDI. Corrosion 53, 617-629 (1997).

[23] J. SÁNCHEZ-MÁRQUEZ, D. ZORRILLA, A. SÁNCHEZ-CORONILLA, D. M. de los SANTOS, J. NAVAS, C. FERNÁNDEZ-LORENZO, R. ALCÁNTARA, J. MARTÍN-CALLEJA, Introducing "UCA-FUKUI" software: reactivity-index calculations. J. Mol. Model. 20, 2492 (2014)

[24] R.G. PARR, W. YANG. J. Am. Chem. Soc. 106, 4049-4050 (1984).

[25] K.F. KHALED. Electrochimica Acta 55, 6523-6532 (2010).

[26] R.I. CASTILLO, L.A.R. MONTALVO, S.P.H. RIVERA. J. Mol. Struct. 877, 1019 (2008).

[27] K.F. KHALED, M.A. AMIN. J. Appl. Electrochem. 38, 1609-1629 (2008).

[28] H.D. LECE, K.C. EMREGUL, O. ATAKOL. Corros. Sci. 50, 1460-1468 (2008).

[29] D.Q. ZHANG, L.X. GAO, G.D. ZHOU. Corros. Sci. 46, 3031-3040 (2004).

[30] F. BENTIS, M. TRAISNEL, H. VEZIN, H.F. HILDEBRAND, M. LAGRENÉE. Corros. Sci. 46, 2781-2792 (2004).

[31] R.T. SANDERSON, Chemical Bonds and Bond Energy. New York, 1976. 
[32] M. RAJENDRAN, A. ALKIYA, P. MUTHUPETCHI, D. DEVAPIRIAM. J. Adv. Sci. Res. 7, 32-37 (2016).

[33] R.G. PEARSON. J. Chem. Ed. 64, 561-567 (1987).

[34] R.G. PEARSON, Coord. Chem. Rev. 100, 403-425 (1990).

[35] R.G. PEAESON. Inorganic Chemistry 27, 734-740 (1988).

[36] I. LUKOVITS, E. KÁLMÁN, F. ZUCCHI. Corrosion 57, 3-8 (2001).

[37] J. ALJOURANI, K. RAEISSI, M. A. GOLOZAR. Corrosion Science 51, 18361843 (2009). 


\section{Abstract}

The objective of this work was to evaluate the efficiency of inhibition the corrosion of two organic molecules derived from benzimidazole, specifically 2-mercaptobenzimidazole (2Mcb) and 2-phenylbenzimidazole (2Fb). The calculations were performed using the Density Functional Theory (DFT) at the $B 3 L Y P$ with $6-311+G(d, p)$ basis set. The quantum parameters correlated with the inhibition efficiency such as the highest occupied molecular orbital energy ( Е $\left._{\text {номо }}\right)$, the lowest unoccupied molecular orbital energy ( $\left.E_{L U M O}\right)$, energy gap $(\Delta E)$, electronegativity $(\chi)$, hardness $(\eta)$, the fractions of electrons transferred $(\Delta N)$, electrophilicity $(\omega)$ and Fukui indices, were calculated. Calculations were performed in aqueous medium in both protonated and non-protonated forms. Theoretical results were compared with experimental data and a good correlation was found between the chemical quantum parameters and the efficiency of inhibition of the molecules.

Key words: Benzimidazole, DFT, Inhibitor, Corrosion.
\end{abstract}

